\title{
Sistema de reconocimiento de patrones de turismo regional aplicando algoritmos de minería de datos
}

\section{Pattern recognition system in regional tourism using data mining}

\author{
NIETO-YAÑEZ, Alma Delia†๋*, HERNÁNDEZ-BÁEZ, Irma Yazmín, LÓPEZ-DÍAZ, Roberto Enrique \\ y ROJAS-SANDOVAL, Daniel
}

Universidad Politécnica del Estado de Morelos

ID $1^{\mathrm{er}}$ Autor: Alma Delia, Nieto-Yañez / ORC ID: 0000-0001-8554-8152, CVU CONACYT ID: 212730

ID $1^{\text {er }}$ Coautor: Irma Yazmín, Hernández-Báez / ORC ID: 0000-0002-3078-9618, CVU CONACYT ID: 212658

ID $2^{\text {do }}$ Coautor: Roberto Enrique, López-Díaz / ORC ID: 0000-0003-1716-6704, CVU CONACYT ID: 212691

ID $3^{\text {er }}$ Coautor: Daniel, Rojas-Sandoval / ORC ID: 0000-0002-7547-2761, CVU CONACYT ID: 43849

DOI: 10.35429/JCT.2019.11.3.1.6

Recibido 12 de Octubre, 2019, Aceptado, 24 de Noviembre, 2019

\section{Resumen}

En México, la Secretaría de Turismo no tiene información suficiente sobre el turismo regional, esto en comparación con el turismo internacional, ya que para éste se realiza un perfil al turista para saber el propósito de su viaje, estadía, transporte utilizado, entre otros datos que permiten identificar mecanismos para mejorar la oferta turística y potencializar la llegada de turistas al país. En contraparte, en el turismo nacional y regional se omiten estos perfiles, por lo tanto, es casi imposible identificar los patrones, un área de oportunidad para obtenerlos, es mediante las redes celulares. El objetivo de este trabajo es identificar patrones de comportamiento de turismo nacional y regional aplicando algoritmos de minería de datos sobre información de conexión de terminales de telefonía celular. La metodología utilizada es KDD utilizando el algoritmo k-means, primero se determina el lugar base de una persona y después si existe turismo en las conexiones de ésta. Con las pruebas realizadas usando perfiles distintos de comportamiento, se logró determinar si una persona realizaba turismo regional y nacional usando medidas de tiempo y distancia entre sus conexiones a una red de telefonía celular.

Minería de datos, Redes de telefonía celular, Turismo nacional y regional

\begin{abstract}
Secretaría de Turismo in Mexico does not have enough information about regional tourism in the country, in comparation with data of international tourism, in which, a tourist profile is obtained to know purpose of the travel, stay, transportation used and another data to allow the identification of mechanisms to improve the tourist offer and, in this way, potentiate the arrival of tourist to the country. On the other hand, to national and regional tourism the profile is omitted, making it difficult to identify patterns of behavior, and is an area of opportunity to obtain them using cell phone networks. The purpose of the presented work is to identify patterns of behavior of national and regional tourism using data mining algorithms to analyze the data of connection of cell phones. The analysis of the information is achieved with KDD methodology in combination with K-means algorithm, first determining the place of residence of a person and next, detecting the connections outside this place. With the executed tests using several patterns of behavior, it was possible to determine if a person carried out national and regional tourism using measures of time and distance between their connections to cell network.
\end{abstract}

Data mining, Cell network, National and regional tourism

Citación: NIETO-YAÑEZ, Alma Delia, HERNÁNDEZ-BÁEZ, Irma Yazmín, LÓPEZ-DÍAZ, Roberto Enrique y ROJASSANDOVAL, Daniel. Sistema de reconocimiento de patrones de turismo regional aplicando algoritmos de minería de datos. Revista de Tecnología Informática. 2019 3-11: 1-6

\footnotetext{
* Correspondencia del Autor (Correo electrónico: anieto@upemor.edu.mx)

$\dagger$ Investigador contribuyendo como primer autor.
} 


\section{Introducción}

La Secretaría de Turismo coloca al turismo como una actividad estratégica de la economía mexicana, tanto por su aportación al producto interno bruto como por los empleos que genera de manera directa e indirecta (SECTUR, 2018).

En el caso del turismo internacional se realiza un perfil para saber el propósito de su viaje, estadía, transporte utilizado, entre otros datos, que permiten identificar mecanismos para mejorar la oferta turística y potencializar la llegada de turistas al país. En contraparte en el turismo nacional y regional se omiten estos perfiles, por lo tanto, es casi imposible identificar los patrones y esto genera que sea complicado analizar la oferta de servicios para la atracción del turismo, así como la infraestructura y la inversión.

El análisis del dinamismo del turismo en México, utilizando indicadores relevantes, obtenidos mediante la identificación de patrones con técnicas de minería de datos ayudaría a incrementar el turismo en el país. La minería de datos es un factor relevante para extraer información de un conjunto de datos y transformarla a una estructura comprensible para un uso posterior. Un área de oportunidad para obtener los patrones, es mediante las redes celulares, que son grandes áreas geográficas divididas en celdas pequeñas, cada celda tiene un identificador y cuando un usuario de telefonía móvil necesita realizar una llamada $u$ otro servicio se conecta a una celda para obtener señal y poder realizar dicho servicio. Cada conexión del usuario y la antena, tiene la hora de inicio y la final, la intensidad y la dirección, a estos datos se aplicarán algoritmos de agrupamiento, que identificarán relaciones que no se podrían derivar lógicamente a través de la observación casual. Para el desarrollo se aplicó la metodología KDD (Knowledge Discovery in Databases).

El objetivo de este trabajo es identificar patrones de comportamiento de turismo nacional y regional aplicando algoritmos de minería de datos sobre datos de conexión de terminales de telefonía celular. La hipótesis planteada es que, con las variables de tiempo y distancia, se podrá determinar tentativamente si existe turismo nacional o regional en una persona.
La estructura del presente artículo es la siguiente: en la sección 1 se presentan algunos de los desarrollos tecnológicos e investigaciones relacionadas a esta investigación; la sección 2 se presenta y describe la metodología utilizada, al igual que las técnicas computacionales utilizadas. En la sección 3, se discuten, las pruebas realizadas y los resultados obtenidos. Finalmente, se concluye el trabajo y se presentan los trabajos futuros.

\section{Trabajos relacionados}

Trabajos recientes han empleado minería de datos para identificar patrones de movilidad usando datos obtenidos mediante telefonía celular. El principal problema en esta información es la oscilación, esto es debido a que las señales de varias torres celulares llegan a un mismo dispositivo y puede parecer que el usuario viaja varios kilómetros en pocos segundos.

En (Ali Bayir, Demirbas, \& Eagle, 2008) se presentan definiciones para capturar patrones y perfiles de movilidad de usuarios de teléfonos celulares y brindan un marco completo, para descubrir los perfiles de usuarios a partir de datos de ubicación basados en celdas. El estudio experimental involucró a 100 personas durante 9 meses. Primero obtienen el tiempo entre dos celdas, el tiempo de transición de dos registros contiguos, el tiempo de la ubicación final normal y el de transición, después de obtener estos datos. Después se agrupan las torres de telefonía celular que ya están etiquetadas por los usuarios, $\mathrm{y}$ en el segundo nivel se manejan las torres restantes sin etiquetar, se asignan a un clúster teniendo en cuenta que podrían ser antenas oscilantes. Enseguida, mediante las rutas de movilidad del usuario como entrada, se realiza un proceso de clúster de antenas.

Posteriormente, se utiliza una versión modificada de la técnica Apriori Secuencial presentada en (Agrawal \& Srikant, 1996) para encontrar patrones. Por último, se agrega información de contexto de tiempo para representar los perfiles de usuario móvil.

Los datos contextuales de tiempo para patrones se especifican en dos dimensiones: Días de la semana y ventanas de tiempo. La agrupación de torres celulares que se realizan en este trabajo reduce el problema de la oscilaciones. 
En (Smoreda, Olteanu-Raimond, \& Couronné, 2013) se describen dos métodos de recopilación de datos de telefonía móvil: localización activa y pasiva. Proponen considerar los datos de ubicación de la red celular como una fuente complementaria útil para la investigación de la movilidad y proporcionar casos de estudios.

También mencionan que a pesar de ser menos precisa que la tecnología GPS la telefonía celular ofrece ventajas: no es necesario implementar una aplicación para recolectar datos, y lo que es más importante que casi toda la población, ya está equipada para un estudio. También ofrece la posibilidad de monitoreo de localización en tiempo real y continuo y si se solicita a los usuarios que validen ciertas ubicaciones se tendrá información más precisa sin invadir mucho la privacidad.

En (Ahas, Raun, \& Tiru, 2014) presentan una forma de determinar el entorno habitual de una persona basado en los datos de posicionamiento móvil, para hacer esto usaron los registros de llamadas del operador de red móvil sobre los horarios y lugares de las actividades de las llamadas.

La base de datos utilizada consistió en las llamadas de teléfonos móviles de los usuarios (con identificación anónima) que permite rastrear el comportamiento espaciotemporal de los turistas. Cada actividad de llamada que se guarda en la base de datos, contiene el número de identificación del teléfono generado al azar, el tiempo de registro y la ubicación marcada.

Usan el método DBSCAN (Density Based Spatial Clustering of Applications with Noises) para medir el entorno habitual con densidad de puntos. En (Suthar, Rajput, \& Gupta, 2013), se presenta un estudio técnico de diferentes enfoques de estos algoritmos de clasificación.

En (Chen, Ma, Susilo, Liu, \& Wang, 2016) se presenta una alternativa para pronosticar la demanda futura de viajes y poder mejorar la inversión del transporte. Para hacer esto usan la información de una base de datos de telefonía móvil generados de forma pasiva, esta se genera como resultado de la comunicación de un teléfono con la red celular mantenida por operadores de telefonía móvil.

\section{Metodología}

El descubrimiento de conocimiento en bases de datos o conocido por sus siglas en inglés KDD (Knowledge Discovery in Databases), es un análisis automático, exploratorio y modelado de grandes repositorios de datos (Fayyad, 1996). KDD es el proceso organizado de identificar patrones válidos, novedosos, útiles $\mathrm{y}$ comprensibles de grandes y conjuntos de datos complejos. La minería de datos es el núcleo del proceso KDD, que involucra la deducción de algoritmos que exploran los datos, desarrollan el modelo y descubren patrones previamente desconocidos (Maimon \& Rokach, 2005). El modelo se usa para entender fenómenos de los datos, análisis y predicción.

A continuación se describen sus diferentes fases:

1. Selección y creación de un conjunto de datos en el que se realizará el descubrimiento. Este proceso es muy importante porque la minería de datos aprende y descubre desde los datos disponibles.

2. Preprocesamiento y limpieza. En esta etapa, se mejora la confiabilidad de los datos. Eso incluye el borrado de datos, como el manejo de valores faltantes y la eliminación de ruido o valores atípicos. Puede involucrar complejos métodos estadísticos o usar un algoritmo de minería de datos en este contexto.

3. Transformación de datos. En esta etapa, se mejoran los datos. Los métodos incluyen la selección y extracción de características y el muestreo de registros, transformación de atributos y transformación funcional.

4. Minería de Datos. Involucra elegir el tipo de técnica y algoritmo empleado para obtener la información. En este trabajo se usó un algoritmo de agrupamiento. El análisis de clúster o clustering es el proceso de partición de un conjunto de datos u observaciones en subconjuntos. Cada subconjunto es un clúster, de modo que los objetos en un clúster son similares entre sí pero disímiles a los objetos en otros grupos. 
El conjunto de clusters resultante de un análisis conglomerado puede denominarse agrupamiento. En este contexto, diferentes métodos de agrupación pueden generar agrupaciones en el mismo conjunto de datos. Los grupos particionados no son realizados por los humanos sino por un algoritmo de agrupamiento. Por lo tanto, la agrupación es útil ya que puede conducir al descubrimiento de grupos previamente desconocidos dentro de los datos (Han , Kamber, \& Pei, 2012). En este trabajo se empleó K-means, el cual es un algoritmo de aprendizaje no supervisado. El objetivo de este algoritmo es encontrar grupos en los datos, con el número de grupos representados por la variable $K$. El algoritmo funciona iterativamente para asignar cada punto de datos a uno de los grupos $K$ en función de las características que se proporcionan. Los puntos de datos se agrupan según la similitud de características.

En lugar de definir grupos antes de mirar los datos, la agrupación le permite buscar y analizar los grupos que se han formado orgánicamente.

Cada centroide de un clúster es una colección de valores de características que definen los grupos resultantes. El examen de los pesos de las características del centroide se puede usar para interpretar cualitativamente el tipo de grupo que representa cada grupo (Trevino, 2016).

5. Evaluación e Interpretación. En esta etapa se evaluan e interpretan los patrones minados (reglas, confiabilidad, etc.) con respecto a los objetivos definidos en el primer paso. Este paso se centra en la comprensibilidad y utilidad del modelo inducido. En este paso, el conocimiento descubierto también está documentado para un uso posterior.

\section{Diseño de la solución}

El diseño de la solución consiste en:

a) Determinar entradas:

Para obtener las entradas diseñó una base de datos y a partir de ella construyeron dos tablas de minado, una para determinar el lugar base y otra para el tipo de turismo.
La tabla para el lugar base incluye: un identificador general, uno del usuario, otro de la conexión, de la antena y por último el tiempo de conexión. Para la tabla del tipo de turismo se le agrega la distancia de la antena a la antena base. Una vez diseñadas se simularon los datos usando un repositorio de antenas registradas en la plataforma OpenCellID (Unwired Labs, 2018), seleccionando 160 antenas de la Ciudad de México.

Con estas antenas se realizó un grafo para poder determinar las adyacencias de cada antena y posteriormente se procede a diseñar dos tipos de perfiles. Los perfiles son importantes, ya que representan el comportamiento de las personas en la vida real, en este caso utilizamos el de un obrero y el de una ama de casa. Para generar los datos de la simulación, es necesario hacer alteraciones en las conexiones de los usuarios, para poder generar turismo, de lo contrario el perfil seguirá la ruta diaria en todas sus conexiones. Las probabilidades para generar una alteración en la conexión de los usuarios reciendo desde el lunes hasta el domingo. En la Figura 1 se muestra el algoritmo empelado para simular los datos de conexión.

\footnotetext{
1. Inicio

2. Determinar el número de antenas que ocupa el usuario por día.

3. Determinar la antena origen(aleatorio entre $1 \mathrm{y}$ el máximo número de antenas).

4. Determinar si se altera la rutina normal.

5. Buscar las adyacencias de la antena anterior.

6. Generar un número para determinar la nueva antena.

7. Si el número de antenas ya se alcanzó ir al paso 8 de lo contrario ir al paso 4.

8. Fin
}

Figura 1 Algoritmo para simulación de datos

b) Determinar lugar base:

Para determinar el lugar base de una persona se toma en cuenta las veces que visitó la antena y el tiempo de conexión en cada antena. Para esto se emplea el algoritmo k-means.

\section{c) Determinar tipo de turismo:}

El tipo de turismo se clasifica dependiendo de la distancia respecto a la antena base: No turismo (si la distancia es menor a $30 \mathrm{~km}$ ), turismo regional (distancia mayor a $30 \mathrm{~km}$ y menor a $100 \mathrm{~km}$ ) y turismo nacional (distancia mayor a $100 \mathrm{~km})$. 
Se emplea k-means, para generar los clusters agregando a la tabla de minado la distancia de cada antena a la antena base determinada anteriormente.

\section{d) Salidas:}

Se realizó una aplicación que recibe los datos generados, el tipo de perfil y el número de clusters y genera gráficas en las que se muestran las agrupaciones generadas.

\section{Pruebas y análisis de resultados}

Con el fin de realizar la experimentación se generaron los datos para un perfil de obrero y otro de ama de casa. Para el perfil del obrero se generaron 2340 conexiones y para el de ama de casa se generaron 4679. Se empleó Weka y una aplicación desarrollada en lenguaje Java y la librería de Weka.

\section{Prueba de lugar base}

El número de grupos para k-means fue determinado con experimentación (ocho para el perfil del obrero y diez para el de ama de casa). Como se aprecia en la Figura 2, el cluster más significativo es el 3 , ya que el usuario pasa el mayor tiempo entre esas antenas. El eje de las $\mathrm{x}$ representa los clusters y el eje de las y el tiempo en días.

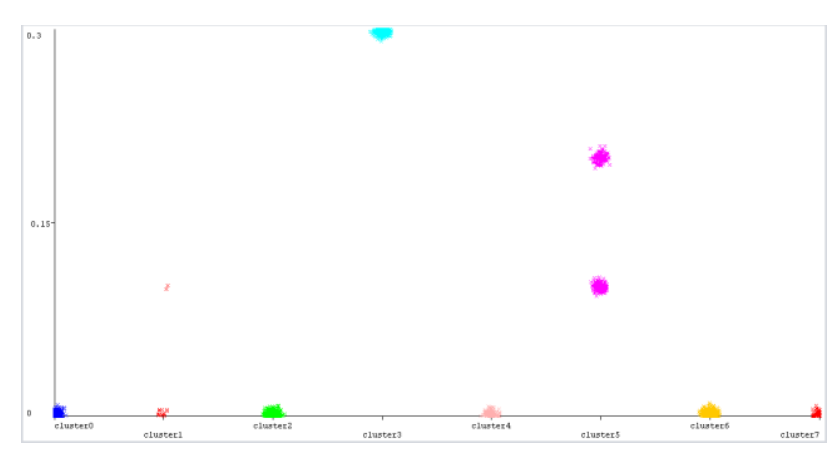

Figura 2 Prueba lugar base del obrero

El cluster 3, es el que agrupa las antenas con las características que se buscan, en este caso el tiempo se mide en días, de tal caso que 0.3 días equivale aproximadamente entre 7 y 8 horas, la estancia del usuario con esa conexión. Por eso se sugiere que ese sea su hogar.

En la Figura 3 se muestra el modo de agrupar del algoritmo, en el eje de las y se tiene el tiempo en horas y en el de las x los clusters.
Como se puede apreciar el cluster 8 es el que tiene una agrupación con los tiempos mayores, siendo estos entre 12 y 14 horas.

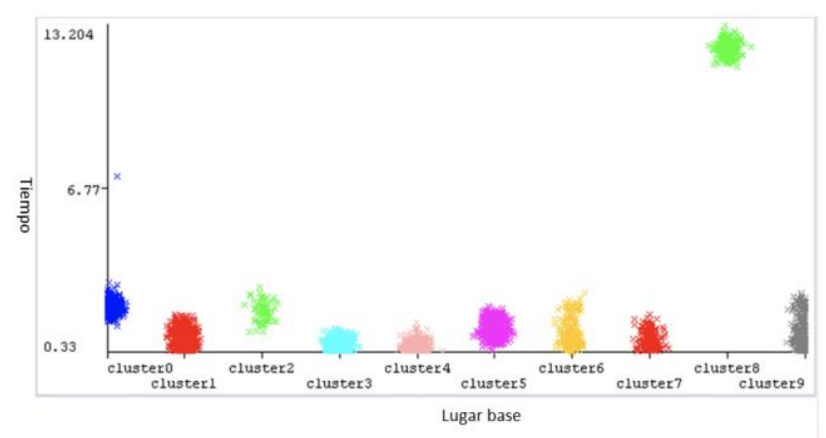

Figura 3 Prueba lugar base de ama de casa

\section{Pruebas turismo regional y nacional}

El objetivo de estas pruebas es determinar si probablemente existe turismo regional y/o nacional en un perfil, para realizar las pruebas es necesario tener ya identificada la antena base, ya que respecto a ella se sacan las distancias con las otras conexiones.

En las pruebas se utilizan como atributos la distancia y el tiempo de conexión y se filtran y sólo se consideran aquellos con un tiempo de conexión significativo. La regla es que el tiempo sea mayor que media hora, para que esa conexión sea significativa.

Para el perfil de obrero después de filtrar quedaron solo 479 datos de conexión. Y para el perfil de ama de casa quedaron 311 datos de conexión. El algoritmo k-means aplicado a los datos filtrado reduce el error y el número de clusters con respecto usar todas las conexiones generadas.

Usando la aplicación desarrollada se puede graficar la agrupación usando distancia y tiempo, distancia lugar y el número de visitas que el usuario realizó al lugar.

En la Figura 4 se muestra la gráfica generada para una instancia de datos de un perfil de ama de casa. Se puede observar los diferentes clusters y con base a la distancia se podría concluir que en este caso si se realizó turismo. 


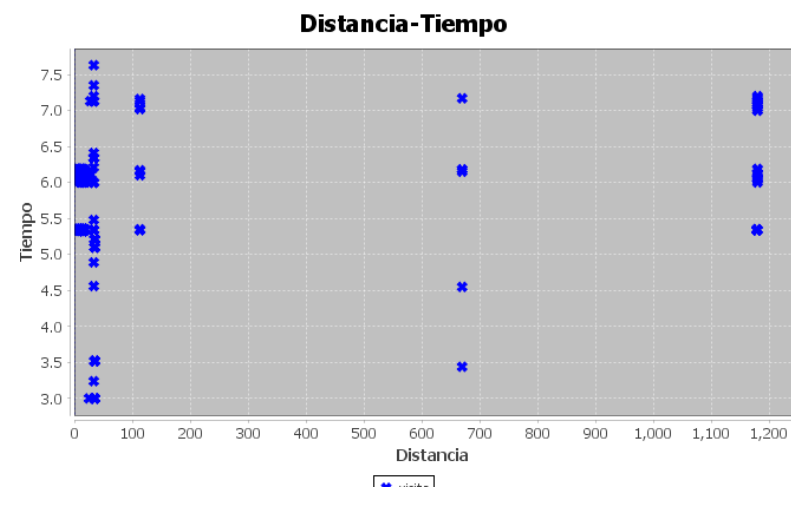

Figura 4 Captura de pantalla de la gráfica distanciatiempo generada por la aplicación

En la Figura 5 se muestra la agrupación de los lugares visitados también de acuerdo a la distancia.

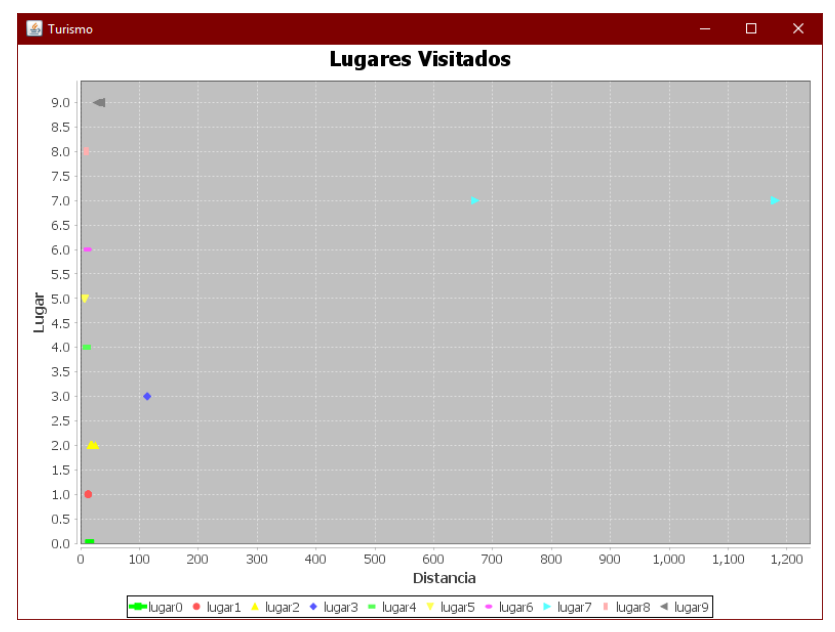

Figura 5 Captura de pantalla de la gráfica de lugares visitados

\section{Conclusiones}

En este trabajo se crearon perfiles y se simularon datos. El proceso para identificar turismo regional y nacional realizado basicamente consta de dos pasos identificar el lugar base y posteriormente usando el lugar base $\mathrm{y}$ las conexiones identificar y agrupar las conexiones que podrían representar turismo regional o nacional, ambos pasos se realizan usando el algoritmo k-means. Usando sólo la distancia y el tiempo se identifica satisfactoriamente si se realiza turismo.

\section{Referencias}

Ali Bayir, M., Demirbas, M., \& Eagle, N. (2008). Mobility Profiler: A Framework for Discovering Mobile User Profiles. Department of Computer Science and Engineering, 2-29.
Agrawal, R., \& Srikant, R. (1996). Mining Sequential Patterns. EDBT, 1-17.

Ahas, R., Raun, J., \& Tiru, M. (2014). Defining usual environment with mobile positioning data. Mobility Lab, 1-4.

Berry, M. (2004). Data Mining Techniques. Indiana: WILEY.

Chen, C., Ma, J., Susilo, Y., Liu, Y., \& Wang, M. (2016). The promises of big data and small data for travel behavior(aka human mobility) analysis. Elsevier, 285-299.

Córdoba Fallas, L. (16 de Junio de 2011). Weka. Obtenido de Weka: http://cormineriadedatos.blogspot.com/2011/06/weka.ht $\mathrm{ml}$

Fayyad, U. P.-S. (1996). From Data Mining to Knowledge Discovery in Databases. Artificial Inteligence Magazine, 17(3), 37-54.

Han , J., Kamber, M., \& Pei, J. (2012). Data Mining Concepts and Techniques. Waltham: Elsevier.

Maimon, O., \& Rokach, L. (2005). DATA MINING AND KNOWLEDGE DISCOVERY HANDBOOK. New York: Springer.

SECTUR. (2018). Nuestro Turismo, el gran motor de la economía nacional (Primera ed.). México: Secretaría de Turismo.

Smoreda, Z., Olteanu-Raimond, A. M., \& Couronné, T. (2013). Spatiotemporal data from mobile phones. Transport Survey Methods, 2040.

Suthar, N., Rajput, I., \& Gupta, V. (May de 2013). A Technical Survey on DBSCAN Clustering Algorithm. International Journal of Scientific \& Engineering Reasearch, 4(5), 17751781.

Trevino, A. (12 de 06 de 2016). Oracle. Obtenido de Introducción a K-means Clustering: https://www.datascience.com/blog/k-meansclustering

Unwired Labs. (2018 de Abril de 2018). OpenCelliD. Obtenido de opencellid.org: https://opencellid.org/\#zoom=16\&lat=37.77889 \&lon=-122.4194 